\title{
SURGICAL PROPHYLAXIS OF RECURRENT STROKE IN PATIENTS WITH PATENT FORAMEN OVALE: A PILOT STUDY
}

\author{
M. Guffi, MD \\ J. Bogousslavsky, $\mathrm{MD}^{\mathrm{b}}$ \\ X. Jeanrenaud, $\mathrm{MD}^{\mathrm{c}}$ \\ G. Devuyst, $\mathrm{MD}^{\mathrm{b}}$ \\ H. Sadeghi, $\mathrm{MD}^{\mathrm{a}}$
}

\begin{abstract}
Prevention of recurrent stroke in adults with patent foramen ovale represents a therapeutic challenge. Antiplatelet or anticoagulant treatment is widely introduced, but its exact indication is not known. In this pilot study, eight men and three women with previous ischemic cerebral events underwent direct surgical closure of the patent foramen ovale. Mean age was 39.4 (from 30 to 58) years. No coexisting cause of stroke was found after extensive investigations, including blood and coagulation tests, echocardiography, 24-hour three-lead electrocardiographic monitoring, extracranial and transcranial Doppler ultrasonography, and cerebral angiography. Criteria for operation also included at least two of the following: atrial septal aneurysm, multiple cerebral infarcts, a history of multiple cerebral events, and Valsalva strain before stroke. Before operation, one patient had two shunts (1 patent foramen ovale, 1 intrapulmonary shunt). No intraoperative or postoperative complications occurred, but a few hours after operation transient arrhythmias developed in two patients without atrial fibrillation, hemodynamic instability, or embolism. During a median follow-up of 12.2 months, no patient had recurrent stroke. All patients prospectively underwent brain magnetic resonance imaging and contrast echocardiography with simultaneous transcranial Doppler ultrasonography. A residual right-to-left interatrial shunt, smaller than the preoperative one, was observed in only one patient, whereas no lesion was seen on magnetic resonance imaging. Our study suggests that surgical closure of patent foramen ovale in patients with presumed paradoxic embolism is safe, with no recurrent stroke in the first year of follow-up. Further studies are needed to evaluate the long-term prognosis of patients with versus without operation and to define the role of operation as an alternative to prolonged antithrombotic treatment. (J Thorac Cardiovasc Surg 1996;112: 260-3)
\end{abstract}

A high prevalence of patent foramen ovale (PFO) in patients with cryptogenic ischemic cerebral infarct has been defined. ${ }^{1-3}$ In these cases, paradoxic embolism is the presumed mechanism, although it is rarely proved. Moreover, the magnitude of the risk of paradoxic embolism in the presence of $\mathrm{PFO}$ has not been determined. In patients with PFO, recur-

From the Divisions of Cardiovascular Surgery, ${ }^{a}$ Neurology, ${ }^{b}$ and Cardiology, ${ }^{c} \mathrm{CHUV}$, University Hospital, Lausanne, Switzerland.

Received for publication August 28, 1995; revisions requested Oct. 18, 1995; revisions received Nov. 17, 1995; accepted for publication Nov. 21, 1995.

Address for reprints: M. Guff, MD, vicolo dell'Orfanotrofio 6, 6900 Lugano, Switzerland.

Copyright (c) 1996 by Mosby-Year Book, Inc.

$0022-5223 / 96 \$ 5.00+0 \quad \mathbf{1 2 / 1 / 7 0 7 9 4}$ rent stroke cannot be completely prevented by antiplatelet therapy, anticoagulation therapy, or transcatheter closure. ${ }^{4,5}$ Concerning the latter procedure, more than $10 \%$ of patients had recurrent transient ischemic attack in a recent study. ${ }^{6}$ In the present study we assessed direct surgical closure of PFO and report the perioperative morbidity and the intermediate follow-up.

\section{Patients and methods}

Patients. Between December 1992 and October 1994, 11 patients ( 8 men, $73 \%$ ) with PFO and previous stroke, from 30 to 58 years old (average age, 39.4 years), were selected for direct surgical closure of PFO. Stroke was defined as an acute, permanent neurologic deficit with a corresponding lesion in a recognized arterial distribution identified by magnetic resonance imaging (MRI) or computed tomography or as an acute focal transient neuro- 
logic deficit without a corresponding lesion on MRI or computed tomography. All subjects were selected on the basis of the protocol reported in Table I, after informed consent was obtained from each patient, respecting the Helsinky principles. Seven patients had at least one of the following thromboembolic risk factors: cigarette smoking ( 3 patients), hypercholesterolemia ( 3 patients), obesity (1 patient), and heredity ( 2 patients). Preoperative transesophageal contrast echocardiography (TEE) with Valsalva maneuver showed positive findings for right-to-left atrial shunting in all patients. Atrial septal aneurysm was demonstrated in three cases, as well as an intrapulmonary shunt in one case, which could not be localized. The criteria used for diagnosis of atrial septal aneurysm included a base width of greater than $1.5 \mathrm{~cm}$ and an excursion beyond the plane of atrial septum of at least 10 mm. ${ }^{7}$ PFO was defined as a natural interatrial channel, which did not close after birth because pressure in the left atrium exceeded that in the right atrium. The maximal size of the orifice reached $16 \mathrm{~mm}$, in accordance with the values reported in previous studies. ${ }^{8}$ One patient had chronic bronchial asthma, treated with terbutaline sulfate.

Operation. All patients underwent the operation via a median sternotomy. Cardiopulmonary bypass (CPB) was instituted with cannulation of the ascending aorta and both venae cavae. Mild hypothermia was achieved, and myocardial protection ensured by infusion of $600 \mathrm{ml}$ hypothermic crystalloid solution into the aortic root and by topical epicardial cooling. The PFO was identified through a right atriotomy. In 10 patients, the PFO was sutured, posterior to the tendon of Todaro, with continuous 4-0 polypropylene suture. Separate stitches were placed in one case. In the patients with atrial septal aneurysm, atrial septal plication was done with the same continuous suture. The atrial incision was closed with 4-0 polypropylene suture and, after deairing, the aortic crossclamp was removed. CPB was discontinued after the patient was warmed to higher than $35^{\circ} \mathrm{C}$ rectal temperature. Before protamine administration, contrast TEE was done, excluding a residual shunt in all patients. The sternal incision was closed in the usual manner.

Postoperative follow-up. Chest roentgenography, electrocardiography, and echocardiography were repeated before hospital discharge. Within a median period of 12.2 months (from 4 to 24.3 months) all patients were seen clinically and underwent ambulatory transcranial Doppler ultrasonography combined with TEE and Valsalva maneuver, which was realized after injection of $10 \mathrm{ml}$ isotonic saline solution containing air microbubbles into a peripheral vein. Chest roentgenography and electrocardiography were also done. Brain MRI was done to detect silent infarcts that might have occurred during follow-up.

\section{Results}

The median time between the initial neurologic event and surgical closure of the PFO was 1.4 months (range 7 days to 89 days). Procedures were done by six surgeons and operative time was 100 to 165 minutes (median, 145 minutes). Crossclamp and CPB median times were, respectively, 16 minutes
Table I. Patient selection criteria

\begin{tabular}{lc}
\hline & $N$ \\
\hline PFO assessed by contrast TEE & 11 \\
Stroke of unknown origin* & 11 \\
At least 2 of following: & \\
Atrial septal aneurysm & 3 \\
Multiple cerebral infarcts & 7 \\
Multiple cerebral events & 9 \\
Valsalva strain before stroke & 3 \\
Age $<59$ yr & 11 \\
\hline
\end{tabular}

${ }^{*}$ No evidence of embolic source at extracranial and transcranial Doppler ultrasonography, MRI, computed tomography, blood and coagulation tests, TEE, and 24-hour electrocardiographic monitoring.

(range 10 to 24 minutes) and 30 minutes (range 25 to 42 minutes). Sinus rhythm reappeared spontaneously in nine patients and after a 10 joule internal electric shock in two. Transient arrhythmias developed in two patients a few hours after the operation (1 nodal rhythm and 1 ventricular tachycardia, which reverted immediately to sinus rhythm after intravenous lidocaine bolus). No atrial fibrillation, hemodynamic instability, or embolic events were seen. Six patients received renal doses of dopamine $(0.5 \mu \mathrm{g} / \mathrm{kg}$ per minute) in the first 12 to 24 hours. Patients left the intensive care unit after a median stay of 19.6 hours (range 8 to 24 hours). Only one patient needed a $600 \mathrm{ml}$ autologous blood transfusion. Total chest drainage ranged from 265 to $670 \mathrm{ml}$ (median, $457 \mathrm{ml}$ ). Chest tubes were removed within 31 to 48 hours (median, 44 hours). Recovery was uneventful in all patients. In particular, there was no pericardial or pleural effusion, no residual intracardiac shunt, no atrioventricular block and no stroke during the 10.5-day median hospital stay (range 8 to 12 days). One patient, known to have psychotic episodes, had behavioral disturbances 2 months after the operation, without evidence of simultaneous thromboembolic events. At 12.2 months no stroke or extracranial embolic event had occurred in any patient, and brain MRI showed no new visible lesion. The patient who had PFO closure with separated stitches has the only residual right-to-left interatrial shunt at the Valsalva maneuver $(15 \mathrm{mi}-$ crobubbles); the preoperative shunt had been larger ( $>20$ bubbles). Although a residual interatrial shunt was excluded by TEE in the patient with intrapulmonary shunt, transcranial Doppler ultrasonography showed late massive contrast passage $(>20$ microbubbles), consistent with this extracardiac shunt. In three patients with correction of atrial septal aneurysm, there was no residual bulging of the interatrial septum. Electrocardiograms and 
chest $x$-ray films showed normal findings in all patients.

\section{Discussion}

A PFO prevalence of $40 \%$ has been reported in young adults with cryptogenic stroke, ${ }^{1}$ but it has been shown that PFO is a risk factor for thromboembolic events in all age groups. ${ }^{9}$ We therefore decided to include older patients (up to 59 years) in our study. However, the tight criteria of our selection protocol enabled us to exclude patients with another potential cause of stroke. Although this has not been demonstrated in a controlled study, we selected patients for operation who had multiple brain events, a history of Valsalva strain, or atrial septal aneurysm because these factors may be associated with higher risk of stroke recurrence than the risk in other patients with stroke and PFO. ${ }^{10,11}$

Currently, the most common treatment to reduce the frequency of recurrent paradoxic emboli is anticoagulation, but prognosis in patients who receive long-term anticoagulation with thromboembolic transient ischemic attacks or stroke is compromised by hemorrhagic complications, mainly cerebral. ${ }^{12-14}$ Buttoned devices constitute another therapeutic alternative, but often must be associated with anticoagulation, especially when stroke recurs. ${ }^{6,15}$ Surgical therapy has the potential of obviating the need for an intracardiac prosthesis and for anticoagulation with its attendant problems of patient compliance and also of excluding recurrent paradoxic embolism. In our series, the only residual shunt was probably a result of a separated stitches technique, which suggests that continuous suture distributes harmoniously the tension on the tissues, decreasing the risk of local rupture. We therefore decided to close PFO systematically with continuous suture. Air embolism is the most serious threat to patients undergoing PFO or atrial septal defect closure and this may be avoided by minimizing intraoperative aspiration of the atrial blood and aspiration of air from the aortic root before electric cardioversion. The few reported deaths have been associated with massive air embolism or perioperative cardiac failure in elderly patients. ${ }^{16}$ Although no deaths occurred in our series and morbidity was minimal, we believe that a prospective randomized trial should be undertaken to evaluate the long-term efficacy of surgical versus medical or interventional approach. The duration of hospitalization was quite long compared with current standards. However, this allowed for careful follow-up. Patients underwent TEE between post- operative days 7 and 10, when the endothelialization process should have been achieved.

Recurrence of stroke after surgical PFO closure may not be linked to paradoxic embolism, but to another cause of stroke. Indeed, in patients with recurrent stroke without residual interatrial shunt or atrial septal aneurysm, a stroke mechanism other than paradoxic embolism must be considered. Furthermore, considering the prevalence of $\mathrm{PFO}$ in the general population (up to $35 \%$ ) and the rather low risk of recurrence in patients with $\mathrm{PFO}$ and stroke, ${ }^{11,13,17}$ it is necessary to identify persons at particularly high risk for paradoxic embolism and stroke recurrence in future studies. Encouraged by the results presented in this paper, we successfully performed surgical PFO closure in 11 additional patients, selected according to the protocol noted herein, who were discharged from the hospital in good clinical condition.

In our experience, surgical therapy for PFO closure in selected patients was safe and no recurrent stroke occurred during follow-up. We suggest that selection criteria used in this pilot study be used in future randomized trials comparing medical and surgical options.

\section{REFERENCES}

1. Lechat PH, Mas JL, Lascault G, et al. Prevalence of patent foramen ovale in patients with stroke. N Engl J Med 1988; 318:1148-52.

2. Webster MWI, Smith HJ, Sharpe DN, et al. Patent foramen ovale in young adult patients. Lancet 1988;2:11-2.

3. Jeanrenaud X, Bogousslavsky J, Payot M, Regli F, Kappenberger $\mathrm{L}$. Patent foramen ovale and cerebral infarct in young patients. Schweiz Med Wochenschr 1990;120:823-9.

4. Matchar JL, McCrory DC, Barnett HJM, Feussner JR. Medical treatment for stroke prevention. Ann Intern Med 1994;121:41-53.

5. Morrison BJ, Landzberg MJ, Newburger JW, Jenkins KJ, Faherty $\mathrm{C}$, Lock JE. Infrequent occurrence of recurrent paradoxical embolism at intermediate follow-up after transcatheter closure of patent foramen ovale. Circulation 1994; 90(Suppl): $\mathbf{2} 237$.

6. Bridges ND, Hellembrand W, Latson L, Filiano J, Newburger JW, Lock JE. Transcatheter closure of patent foramen ovale after presumed paradoxical embolism. Circulation 1992;86: 1902-8.

7. Pearson AC, Nagelhout D, Castello R, Gomez C, Labovitz A. Atrial septal aneurysm and stroke: a transesophageal echocardiographic study. J Am Coll Cardiol 1985;6:1370-2.

8. Hagen PT, Scholz DG, Edwards WD. Incidence and size of patent foramen ovale during the first 10 decades of life: an autopsy study of 965 normal hearts. Mayo Clin Proc 1984;59: $17-20$.

9. De Belder MA, Tourikis L, Leech G, Camm AJ. Risk of patent foramen ovale for thromboembolic events in all age groups. Am J Cardiol 1992;69:1316-20. 
10. Cabanes L, Mas JL, Cohen A, et al. Atrial septal aneurysm and patent foramen ovale as risk factors for cryptogenic stroke in patients less than 55 years of age: a study using transesophageal echocardiography. Stroke 1993;24:1865-73.

11. Zuber M, Mas JL. Risk of recurrent stroke in patients with atrial septal aneurysm or patent foramen ovale: a French multicentric study. Cerebrovasc Dis 1994;4:247.

12. Bogousslavsky J, Regli F. Anticoagulant-induced intracerebral bleeding in brain ischemia. Acta Neurol Scand 1985;71: 464-71.

13. Garazi S, Bogousslavsky J, Jeanrenaud X, van Melle G. Abnormal interatrial septum with stroke: recurrence risk and late prognosis in 103 patients. Cerebrovasc Dis 1994;4:247.
14. Mas JL. Secondary prevention of cerebral embolism of cardiac origin. J Mal Vasc 1993;18:283-92.

15. Rao PS, Wilson AD, Chopra PS. Transcatheter closure of atrial septal defect by "buttoned" devices. Am J Cardiol 1992;69:1056-61.

16. Kirklin JW, Barratt-Boyes BG. Atrial septal defect and partial anomalous venous connection. In: Kirklin JW, Barratt-Boyes BG, eds. Cardiac surgery. 2nd ed. New York: Churchill, Livingstone, 1993:634-5.

17. Homma S, Di Tullio MR, Sacco RL, Mihalatos D, Li Mandri G, Mohr JP. Characteristics of patent foramen ovale associated with cryptogenic stroke: a biplane transesophageal echocardiographic study. Stroke 1994;25:582-6.

\section{Notice from The Thoracic Surgery Foundation for Research and Education}

Information and applications for 1996 Research Grants, Research Fellowships, and Career Development Awards are now available. The deadline for receipt of completed applications is October 14, 1996. Please contact Amy Hedmark to request an application or further information.

The Thoracic Surgery Foundation for Research and Education, 401 North Michigan Ave., Chicago, IL 60611; phone, 312-644-6610; fax, 312-527-6635. 